\title{
Estrategias de inclusión-exclusión de la ciudad colombiana autoproducida mediante políticas de reasentamiento barrial
}

Stratégies d'inclusion-exclusion de la ville colombienne auto-construite grâce aux politiques de réaménagement des quartiers

Strategies for inclusion-exclusion in the Colombian city that are self-produced through a policy of resettled neighborhoods

\section{Carlos Alberto Torres Tovar y Solanyi Robles Joya}

\section{OpenEdition}

Journals

Edición electrónica

URL: http://journals.openedition.org/bifea/5975

DOI: $10.4000 /$ bifea.5975

ISSN: 2076-5827

Editor

Institut Français d'Études Andines

Edición impresa

Fecha de publicación: 1 diciembre 2014

Paginación: 587-609

ISSN: 0303-7495

Referencia electrónica

Carlos Alberto Torres Tovar y Solanyi Robles Joya, « Estrategias de inclusión-exclusión de la ciudad colombiana autoproducida mediante políticas de reasentamiento barrial », Bulletin de l'Institut français d'études andines [En línea], 43 (3) | 2014, Publicado el 08 diciembre 2014, consultado el 05 noviembre 2020. URL : http://journals.openedition.org/bifea/5975 ; DOI : https://doi.org/10.4000/bifea.5975

Les contenus du Bulletin de l'Institut français d'études andines sont mis à disposition selon les termes de la licence Creative Commons Attribution - Pas d'Utilisation Commerciale - Pas de Modification 4.0 International. 


\title{
Estrategias de inclusión-exclusión de la ciudad colombiana autoproducida mediante políticas de reasentamiento barrial ${ }^{*}$
}

\author{
Carlos Alberto Torres Tovar** \\ Solanyi Robles Joya ${ }^{* * *}$
}

\begin{abstract}
Resumen
Se abordan algunas percepciones y respuestas a las estrategias de inclusión-exclusión de la ciudad autoproducida mediante políticas de reasentamiento barrial. El texto se centra en observar el desarrollo de los procesos de reasentamiento efectuados en Bogotá. Estos han buscado reducir la vulnerabilidad física de los asentamientos mediante el Mejoramiento Integral de Barrios — MIB - , el traslado de los hogares localizados en zonas de riesgo no mitigable y la recuperación de las rondas de los cuerpos de agua. Para ello se analizan las formas dominantes de exclusión en la ciudad, la producción de la informalidad y las búsquedas por la inclusión, así como las políticas de reasentamiento barrial.
\end{abstract}

* Este artículo se basa en los hallazgos de dos investigaciones: la primera «Estructuración de un modelo base para políticas públicas nacional y locales orientada al mejoramiento barrial y urbano en ciudades colombianas: una estrategia para la superación de la informalidad y la pobreza urbana», adelantada por el Grupo de Investigación «Procesos urbanos en hábitat, vivienda e informalidad» de la Universidad Nacional de Colombia con la cofinanciación de Colciencias durante los años 20112012. La segunda corresponde al trabajo de investigación para la producción de la tesis de Magister en Hábitat en la Universidad Nacional de Colombia de la Arq. Solanyi Robles-Joya denominada «Impactos del reasentamiento por vulnerabilidad en áreas de alto riesgo en Bogotá. 1991-2005», culminada en 2008.

** Arquitecto, Magíster y Doctor en Arquitectura y Ciudad y en Urbanismo. Profesor Asociado Universidad Nacional de Colombia. Escuela de Arquitectura y Urbanismo. Investigador Senior (IS) Colciencias. Líder del Grupo de Investigación «Procesos Urbanos en Hábitat, Vivienda e Informalidad». Dirección Carrera 30 n. ${ }^{\circ}$ 45-03. Edificio SINDU. Oficina 106. Bogotá. E-mail: catorrest@unal.edu.co

***Arquitecta, Magíster en Hábitat. Profesora Universidad Colegio Mayor de Cundinamarca y Universidad la Salle. Miembro del Grupo de Investigación «Procesos Urbanos en Hábitat, Vivienda e Informalidad». Dirección Carrera 30 n. ${ }^{\circ}$ 45-03. Edificio SINDU. Oficina 106. Bogotá. E-mail: sroblesj@unal.edu.co 
Palabras clave: informalidad urbana, reasentamiento barrial, agentes urbanos, vivienda, pobreza, neoliberalismo, Bogotá

\title{
Stratégies d'inclusion-exclusion de la ville colombienne auto- construite grâce aux politiques de réaménagement des quartiers
}

\section{Résumé}

Sont ici abordées quelques suggestions et réponses aux stratégies d'inclusion-exclusion de la ville autoconstruite à travers des politiques de réaménagement de quartiers. Le texte cherche à observer les processus de réaménagement effectués à Bogotá. Ces processus ont cherché à réduire la vulnérabilité physique des quartiers à travers I'Amélioration Intégrale de Quartiers —MIB—, le relogement des foyers placés dans des zones à haut risque et la récupération des berges des cours d'eau.

Des éléments comme les formes dominantes d'exclusion, l'informalité urbaine, la recherche de l'inclusion sociale et les politiques de réaménagement des quartiers, sont analysés dans cet article.

Mots-clés : informalité urbaine, réaménagement des quartiers, agents urbains, logement, pauvreté, néolibéralisme, Bogotá

\section{Strategies for inclusion-exclusion in the Colombian city that are self- produced through a policy of resettled neighborhoods}

\begin{abstract}
This article addresses some perceptions and answers to the strategies of inclusion-exclusion of the city that are produced through neighborhood resettlement policies. The article focuses on observations made concerning the development of the resettlement process carried out in Bogotá. These have sought to reduce the physical vulnerability of settlements through Integral Neighborhood Improvement program (MIB, in Spanish), that involves moving households located in areas at high risk that cannot be mitigated. To do this we analyze the dominant forms of exclusion in the city, the production of informality and search for inclusion, such as neighborhood resettlement policies.
\end{abstract}

Key words: urban informality, neighborhood resettlement, urban agents, housing, poverty, neoliberalism, Bogotá

\section{INTRODUCCIÓN}

Construir miradas sobre los problemas urbanos presentes es fundamental para entender las sociedades actuales y futuras. Por ello, este texto no pretende hacer un examen unívoco, sino generar interrogantes que permitan acercarse a otras percepciones y respuestas a una cuestión tan compleja como son los conflictos de la ciudad colombiana y en ella las estrategias de inclusión-exclusión de la ciudad autoproducida mediante políticas y programas de reasentamiento barrial —PRB—. Así, este texto se centra en observar el desarrollo de los procesos de reasentamiento efectuado por instituciones públicas de Bogotá, como entidades que buscan reducir la vulnerabilidad física de los asentamientos de origen informal a través de diferentes intervenciones, como el mejoramiento integral de barrios — MIB - el traslado de los hogares localizados en zonas de riesgo no mitigable y la recuperación de rondas de ríos y cuerpos de agua. 
Estas iniciativas aunque son positivas no son suficientes, ya que las entidades encargadas de los PRB han adelantado procesos que solo apuntan a acompañar a la población durante el transcurso del mismo, sin que exista un seguimiento de los impactos que ello ocasiona. Lo que se intenta prioritariamente desde estos procesos es garantizar el derecho a la vida dada la localización de población en zonas de riesgo no mitigable. Sin embargo, simultáneamente estos PRB terminan vulnerando otros derechos al generar la ruptura de las redes sociales, las dinámicas económicas y las prácticas culturales que le daban soporte a las comunidades barriales.

Frente a este problema y mediante la presentación de dos casos de estudio se identifican los impactos ocasionados por los PRB, formulando, a su vez, algunas consideraciones para la construcción de una adecuada política pública de reasentamiento que se constituya a su vez en una estrategia de inclusión de esta ciudad colombiana autoproducida.

\section{ESPACIO URBANO Y RESIDENCIAL DE LA POBLACIÓN DE BAJOS INGRESOS EN BOGOTÁ}

Iniciando el siglo XXI, es claro que las ciudades latinoamericanas han continuado creciendo de manera acelerada en las últimas décadas, extendiendo su tamaño y ampliando su población, sin que aún logren consolidarse, presentando altos niveles de aglomeración que han ido de la mano de un desarrollo desequilibrado e inequitativo de la ciudad, con enormes desigualdades en todos los órdenes, aumentando así las condiciones de exclusión.

Así Latinoamérica, profundiza el modelo neoliberal que se refleja en todos los ámbitos de la vida de la ciudad, tanto los públicos, privados como comunitarios. Modelo que genera a su vez una visión territorial que se construye espacializando un modo de producción que basa todo su crecimiento y progreso en las fuerzas del mercado.

Ciudades en las que el crecimiento urbano se ha configurado mediante la superposición prioritaria de dos modos básicos de producción de ciudad, el formal e informal, los cuales basan su racionalidad en formas diferenciadas de mercado, incidiendo en la habitabilidad y en las condiciones y calidad de vida de la mayoría de la población urbana (Torres, 2009a).

Este modelo de ciudad parte fundamentalmente por reconocer un conjunto de población presente en el territorio, disponible para poder atender las distintas actividades y las distintas lógicas que establece el mercado. Por lo tanto, dentro de esa perspectiva el problema del modelo no se centra en resolverle la problemática ni las necesidades al conjunto de la población (en ello los problemas derivados de la localización en áreas vulnerables y en riesgo de la población presente en asentamientos autoproducidos); el modelo se centra fundamentalmente en disponer de los recursos que están en ese territorio, para poder fortalecer 
el modelo, indistintamente de que todos puedan ser beneficiados o no (Brand, 2009: 273).

Por lo tanto, el modelo de ciudad y su modelo de desarrollo están basados en políticas de enfoque neoliberal, lo cual ha significado en las dos últimas décadas, el desmonte de la búsqueda del denominado estado bienestar a cambio de un estado regulador de mercado. Modelo que ha efectuado la transferencia de la responsabilidad pública al sector privado en materia de servicios públicos (privatización de las empresas de energía, agua potable, telecomunicaciones, gas natural, aseo, y transporte), en materia de servicios sociales (imposición de un modelo de privatización de la salud, la educación y el bienestar social), y en la venta de todos los activos públicos con el pretexto de reinversión para la atención de las necesidades sociales. Este patrón configura preferentemente estrategias de exclusión antes que de inclusión, siendo la principal excluida, la ciudad autoproducida (Torres, 2005: 118-119).

Bajo este marco, los resultados de la investigación adelantada señalan que son cinco los aspectos que son fundamentales en el análisis de los problemas presentes en el espacio urbano y residencial de Bogotá: el aumento sostenido de la pobreza y la miseria; las dinámicas económicas de la población de bajos ingresos - $\mathrm{PBI}$ - ; las condiciones habitacionales de los hogares de la PBI a las que se suman el déficit habitacional, tanto cuantitativo como cualitativo; la llegada de población en condición de desplazamiento forzado derivada del conflicto social y armado que afronta Colombia; y, por último, la profundización de la segregación socioespacial.

Como parte de los rasgos generales de la ciudad (SDP, 2013), dos asuntos son de destacar. El primero, la configuración barrio a barrio con la que se ha construido Bogotá, lo cual permite a su vez explicar el modelo de producción de la ciudad y la forma como se habilita el suelo urbano (Hataya et al., 1994). El segundo, la identificación de los principales agentes urbanos intervinientes en la producción, transformación y consumo de suelo y vivienda en Bogotá (Torres et al., 2011). En estos rasgos generales, se reconocen los problemas derivados del crecimiento demográfico y espacial de la ciudad, su estructura política y el sistema político administrativo, la estructura de la planeación urbana y sus determinantes (gestión urbana, planificación y ocupación territorial) sobre los cuales se configura el modelo de ciudad neoliberal; la estructura social, la estructura económica y las problemáticas urbanas en materia de urbanización.

Así mismo, Bogotá presenta un aumento sostenido de la pobreza y miseria, lo cual es observable a partir de las dinámicas económicas que adopta la PBI (economía informal, rebusque), la profundización de la segregación socioespacial, los impactos en la población derivados de la estratificación socioeconómica y las repercusiones sobre las condiciones habitacionales. En Bogotá, la pobreza se cuantifica utilizando diferentes metodologías que son contradictorias ya que unas indican una disminución vertiginosa en los últimos años. Tal es así que para 2012, solo el $11,6 \%$ del total de la población estaba en condición de pobreza y el 2\% se encontraba en condición de pobreza extrema (SDP, 2013). En tanto otras muestran 
que el 23,8\% de la PBI está bajo la Línea de Pobreza (1 763716 habitantes) y el 3,4\% se encuentra bajo la Línea de Indigencia (251 960 habitantes) (PNUD, 2008). A su vez la concentración de la riqueza medida por el Coeficiente GINI, para el mismo año, se sitúa en 0,497 siendo alto (SDP, 2013). Estas estadísticas no logran evidenciar la magnitud de la problemática y la realidad que se vive en los territorios autoproducidos.

Los asuntos relacionados con el déficit habitacional, tanto cuantitativos como cualitativos, muestran cómo la ciudad no ha logrado avances significativos en esta materia, ya que los déficits presentes a comienzos de los años 1990 aún se mantienen. A ello se suma la poca oferta de suelo disponible para urbanizar y, entre estas, la necesaria para atender las demandas acumuladas de vivienda social (para al año 2010 existían aún 6600 hectáreas de suelo vacante urbanizable en manos del mercado (Torres et al., 2011). La aparente «escasez» en materia de suelo para vivienda de interés social — VIS - que impide, a decir de los promotoresconstructores involucrados en esta parte del sector inmobiliario, el desarrollo de este tipo de vivienda se debe a la especulación y acaparamiento del mismo por parte de los «suelo-tenientes» urbanos y otros agentes urbanos privados cuyo interés se centra en proyectos de alta rentabilidad financiera y no en la provisión de viviendas adecuadas para la PBI.

Se reconoce que más del $50 \%$ de las viviendas de los habitantes de Bogotá se encontraban clasificados en 2009 en los dos estratos socioeconómicos más bajos, 1 y 2, localizándose mayoritariamente en la periferia de la ciudad, distantes de los principales centros de generación de empleo y de la mayor oferta de bienes y servicios de la ciudad. En tanto que menos del 5\% de la población se encontraba ubicada en los dos estratos más altos, 5 y 6 (SDP, 2013).

De igual manera, un fenómeno persistente y que se continua presentando, es el alto número de población en condición de desplazamiento forzado que llega a Bogotá, que si bien se remonta a la denominada época de la violencia en los años 1950, son los procesos de más reciente aparición desde mediados de la década de 1980 y sobre todo en la de 1990, por efectos del conflicto social y armado interno que se vive en Colombia, lo que genera este fenómeno de atracción poblacional a la ciudad. El número de desplazados por esta violencia generada y la agudización del conflicto en Colombia, da cuenta que entre 1985 y 2013 han Ilegado a Bogotá más de 795364 de desplazados (10\% de los habitantes de Bogotá), principalmente a los cinturones de miseria en los barrios de origen informal (Codhes, 2014), correspondiendo al 15,3\% del total nacional de población en condición de desplazamiento forzado. Esta es una realidad que impacta a la ciudad y de manera particular a la vivienda, ya que esta población no retorna a sus lugares de origen y requiere solucionar el acceso a la vivienda1.

1 El 75\% de la población que llega a las ciudades producto del desplazamiento interno ya tenía resuelto el problema de la vivienda en términos de acceso a la misma, ya fuese en posesión o por propiedad (Torres, 2009b). 
Por otra parte, la organización socioespacial de la ciudad es segregada, tanto espacial (segregación residencial) como socioeconómicamente, con muy escazas mezclas entre ricos y pobres. Presenta una mayor segregación entre estos tipos de población, con una mala distribución del espacio público, de los equipamientos colectivos, enormes distancias, tiempos y costos de desplazamiento urbano, entre otros bienes y servicios urbanos. Esta segregación se observa en la forma como se distribuye la población y con ella la vivienda en la ciudad, complementada con el procedimiento administrativo de la estratificación socioeconómica² que segmenta la población en seis grupos. Segregación que incluso se marca entre las prácticas culturales de los ricos al norte y los pobres al sur. Como señala la SDP,

... la segregación en Bogotá (...): es elevada, es secular y tiende a reproducirse (SDP, 2007: 11).

Este alto nivel de segregación socioespacial revela dos procesos de producción del espacio urbano determinados territorialmente por la ciudad formal (planificada, reglamentada y autónoma), y la ciudad informal o «ilegal» (ver el mapa de la segregación realizado a partir de índices dotacionales en el Documento Técnico de Soporte del POT de 20133).

Un paréntesis necesario permite argumentar, desde una perspectiva concreta, un cuestionamiento de la dualidad de la ciudad latinoamericana (ciudad formal/ ciudad informal-ciudad autoproducida). Por un lado, ambas «ciudades» (formal e informal) son el espacio residencial de la PBI. Por otro, más allá de la relación dialéctica en el plano económico entre ambas «ciudades», en relación a la producción de vivienda, está demostrado que son realidades que se entrecruzan y deslizan de manera permanente, con diferentes intensidades y matices.

En el caso de la ciudad informal o autoproducida, la existencia y crecimiento de este tipo de asentamientos constituyen la expresión más clara de la pobreza, desigualdad, segregación, el crecimiento urbano incompleto, la deficiencia en la oferta de suelo y vivienda social, y el débil proceso de ordenamiento territorial. Demuestra, a su vez, que la exclusión social, económica y, por qué no, política e ideológica se manifiesta en la estructura del espacio urbano y residencial (exclusión espacial) e impacta de forma negativa la calidad de vida de la PBI. De hecho, la llamada ciudad informal constituye, a 2013, más del 20,9\% del total del área urbanizada de Bogotá y conforma 1653 (1541 legalizados y 112 en proceso) ${ }^{4}$ de los 2250 barrios de la capital colombiana (ver el mapa de Acumulado de los asentamientos de origen informal en el Documento Técnico de Soporte del POT de 20135).

2 La clasificación depende de la condición físico espacial de su localización y afecta a la forma y los costos con que la PBI puede acceder a los bienes y servicios urbanos, particularmente los servicios públicos domiciliarios y los servicios colectivos.

3 SDP, 2013: 629.

4 Ocupan aproximadamente 8059 ha, se calculan 427681 lotes y una población estimada en 2309477 habitantes, es decir, el 30,9\% de la población residente en Bogotá (Alcaldía Mayor de Bogotá-AMB, 2013).

5 SDP, 2013: 299. 
A ello habría que agregar los procesos recientes de densificación en áreas residenciales y la aparición de nuevos procesos de ocupación del territorio urbano a través de fenómenos como la redensificación urbana — tanto en áreas de origen formal como en áreas de origen informal—-, procesos de gentrificación, acciones de promoción inmobiliaria a partir de intervenciones de renovación urbana, todos ellos como acciones rentísticas que excluyen a la PBI.

Se puede afirmar, entonces, que el espacio urbano y residencial de la $\mathrm{PBI}$ en Bogotá es deficitario, incompleto y que estimula la segregación y la exclusión social, más aún cuando no son solo las prácticas del mercado las que lo generan sino que a su vez son los impactos derivados de las políticas públicas.

\section{LAS POLÍTICAS DE REASENTAMIENTO BARRIAL COMO PRÁCTICA GENERAL}

Al reflexionar sobre las problemáticas del hábitat y la vivienda en Colombia se evidencia que en la configuración de sus ciudades aparecen fenómenos marcados por la segregación y la exclusión de las mayorías pobres, que ha dado como resultado la irregularidad de su permanencia en los territorios que habitan y la limitación de las garantías para la construcción de un hábitat digno.

Estas ciudades tienden a crecer ilimitadamente bajo lógicas que combinan la formalidad con la informalidad, siendo esta última la dinámica preponderante. En las urbes se aglutinan diariamente cientos de personas que buscan mejorar su calidad de vida, encontrar opciones laborales y, en algunos casos, mantenerse vivos en un país donde es preciso huir para existir.

Llegan a ubicarse en lotes (parcelas) que no cuentan con las condiciones urbanísticas y ambientales adecuadas, como tampoco un entorno adecuado con equipamientos, bienes y servicios urbanos que permitan soportar estos nuevos hábitats. La invasión de terrenos no urbanizables se convierte, entonces, en la constante y no en la excepción de nuestras ciudades. Constante que, a su vez, es causa y consecuencia de las limitaciones institucionales para la generación de políticas públicas de hábitat y vivienda capaces de atender la realidad nacional, más allá de los flujos del mercado y el interés del sector privado (Torres, 2012).

En este marco, los PRB aparecen como una de las herramientas de política pública para la reorganización del contexto físico y social de las ciudades. Su configuración conceptual ha dependido de la influencia de distintas variables, entre las cuales resalta, para el caso colombiano, el fenómeno del desplazamiento forzado, ante el cual el desarrollo de los procesos, programas y políticas de reasentamiento de población en una condición de emergencia ha sido creciente.

Las causas involucradas con el reasentamiento, están relacionadas con las condiciones de localización de la población en áreas con riesgo no mitigable, ya sea por fenómenos naturales como inundación, cuando se habita un territorio con una fuente hídrica (ver cartografía 04 «Inundación por desbordamiento» del Decreto 364 de 2013); por remoción en masa, cuando se vive en las laderas de 
una montaña y aumenta la vulnerabilidad con la construcción en zonas de cantera (ver cartografía 05 «Remoción en masa» del Decreto 364 de 2013), y, por último, por falla sísmica, en lugares geológicamente vulnerables.

Las instituciones involucradas con los procesos de reasentamiento, en sus distintas formas, en Bogotá han sido: el instituto de desarrollo urbano — IDU—, la dirección de prevención y atención de emergencias _ DPAE—6, la empresa de acueducto de Bogotá —EAAB — la caja de vivienda popular - CVP - y la secretaria distrital de ambiente —SDA — . Ellas han adelantado el diseño y la ejecución de políticas que siguen manteniendo un marcado énfasis en la dimensión físico espacial del problema (fig. 1)

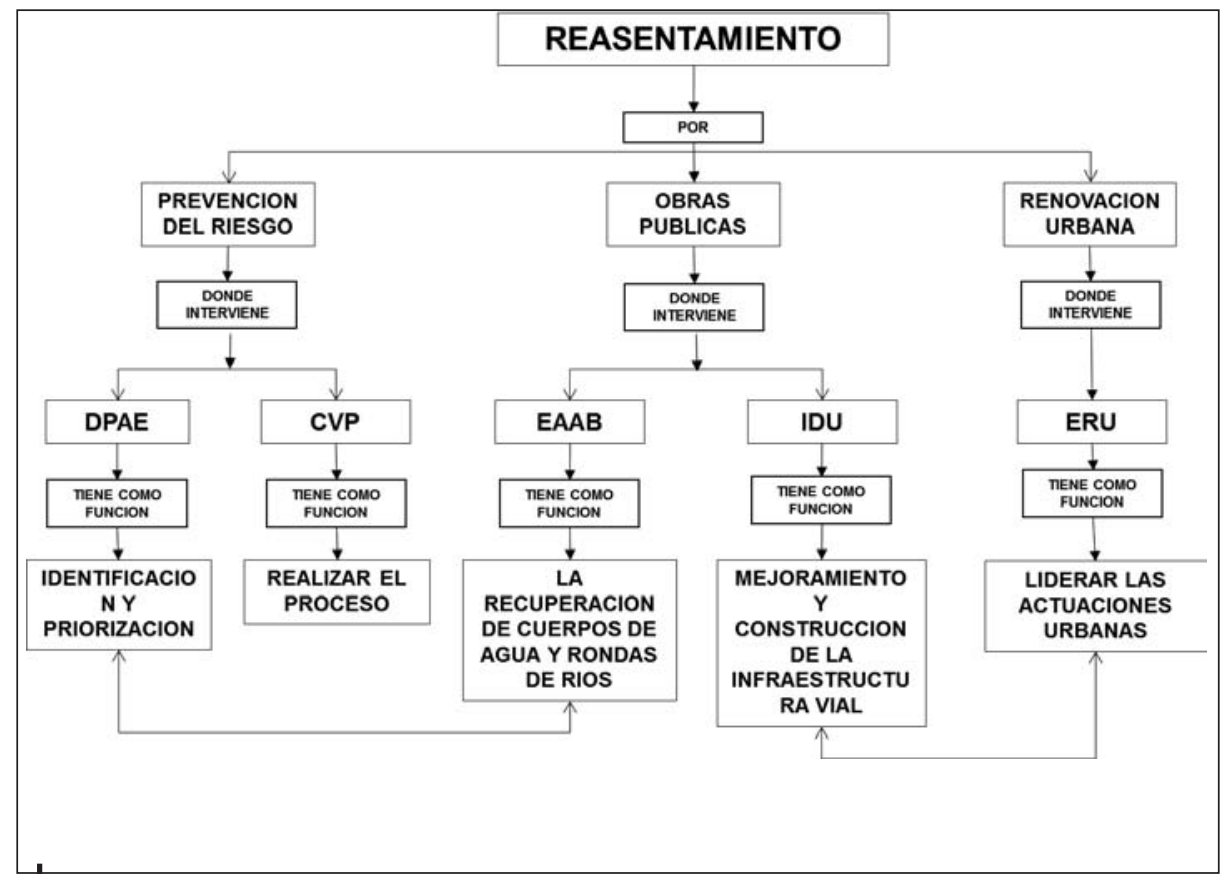

Figura 1 - Entidades distritales en relación al reasentamiento

Fuente: Robles, 2014

Sin embargo, y a pesar de los constantes ajustes institucionales las entidades de Bogotá encargadas de los PRB, han generado la ruptura de las redes sociales, económicas y culturales que le daban soporte a estas poblaciones, ya que asumen que en estos procesos, los factores como las fuentes de trabajo, educación y salud, se dan por adquiridos en el momento del reasentamiento.

6 En 2014 existe también el instituto distrital de gestión de riesgo y cambio climático —IDIGER—, y el fondo de prevención y atención de emergencias -FOPAE-. 
En este sentido es preciso señalar que los procesos de reasentamiento no solo se deben limitar a mitigar los peligros derivados de las condiciones físicas de los asentamientos, sino que deben, también, asumir de forma integral el mejoramiento de las condiciones de vida de la población y asegurar su inclusión en la ciudad. Por lo tanto, lo que se propone es ampliar el lente de observación para ubicar las relaciones entre dichos procesos y políticas, con el cumplimiento de los derechos humanos, el derecho a la vivienda y, en particular, el derecho a la ciudad.

Así pues, se pretende abordar el reasentamiento desde una perspectiva de derechos, poniendo a los habitantes en el centro de la problemática considerándolos agentes activos y conscientes de la construcción social del territorio; sin embargo, no se pretende caer en radicalismos que ignoran las relaciones sistémicas que se construyen con la naturaleza y las significaciones históricas presentes en cada contexto. Se requiere una mirada integradora que valore la importancia de los elementos que constituyen y definen el hábitat.

Para el caso de Bogotá se establecieron cuatro tipos básicos de reasentamiento7: por obra pública, por procesos de renovación urbana, por prevención del riesgo (altas condiciones de vulnerabilidad), y por desastre (procesos de remoción en masa, inundaciones, fallas geotécnicas y/o geológicas). La manera como las instituciones distritales atienden estas demandas se observa en la figura 1.

Los procesos de reasentamiento por obra pública y por recuperación de rondas de río y cuerpos de agua, se presentan por el desarrollo de obras en las cuales se busca el mejoramiento de las condiciones urbanísticas de la ciudad. Procesos que han implicado el necesario reasentamiento de pobladores ubicados en las zonas donde dichas obras se llevan a cabo.

Las obras son realizadas por entidades descentralizadas, en el caso de Bogotá por el IDU8. Dentro de las funciones adjudicadas a esta entidad está la de adquirir predios para el desarrollo de las obras lo que genera desplazamiento involuntario de la población afectada por los proyectos99, razón por la cual la entidad genera mecanismos que permiten la negociación con los propietarios o poseedores de los predios necesitados.

Así como el reasentamiento por «obra pública» es asumido por el IDU, el que se origina por la recuperación de rondas de río y cuerpos de agua es responsabilidad de la EAAB 10 .

Basado en la Investigación «Impactos del reasentamiento por vulnerabilidad en áreas de alto riesgo en Bogotá. 1991-2005», adelantada por Solanyi Robles en el marco de la Maestría en Hábitat (Robles, 2008).

8 Creado mediante el artículo 2 del Acuerdo 19 de 1972. Expedido por el Concejo de Bogotá, con la finalidad de ejecutar obras públicas de desarrollo urbanístico. Con el tiempo se convirtió en la única entidad encargada del desarrollo de este tipo de proyectos en la ciudad.

9 «Debido a su papel fundamental como ejecutor de obras públicas es una de las entidades que genera mayor traslado involuntario de población derivado de la adquisición de los predios requeridos para la realización de las obras» (Arrieta, 2005: 47).

10 Empresa comercial del Distrito Capital creada en 1955 por el Acuerdo 105 del Concejo de la ciudad, la cual tiene como objeto «(...) la prestación de los servicios públicos esenciales domiciliarios de 
La incorporación del tema del reasentamiento a los procesos de la EAAB, inicia en 1994 a partir del empréstito con el Banco Mundial, el cual dispuso dentro de sus obligaciones el inicio de PRB para reducir el impacto del desplazamiento involuntario en la población afectada al efectuar las obras.

Dicha incorporación se llevó a cabo a través de una resolución interna en la cual se establecieron los criterios para el pago de las compensaciones a los hogares reasentados11 (EAAB, 2006), limitándose a ello el alcance desarrollado.

El Reasentamiento por procesos de Renovación Urbana ${ }^{12}$ es asignado a la ERU13, la cual tiene como objetivo gestionar, liderar, promover y coordinar, mediante sistemas de cooperación, integración inmobiliaria o reajuste de tierras, la ejecución de actuaciones urbanas integrales para la recuperación y transformación de sectores deteriorados del suelo urbano, mediante programas de renovación y redesarrollo urbano, y para el desarrollo de proyectos estratégicos en suelo urbano y de expansión con el fin de mejorar la competitividad de la ciudad y la calidad de vida de sus habitantes (Acuerdo 33, 1999).

La renovación urbana ${ }^{14}$ es definida por el artículo 363 del POT (Modalidades y áreas de aplicación del tratamiento de renovación urbana), a través de las modalidades de reactivación y redesarrollo así: enfocándose el redesarrollo a los $«$... sectores donde se requiere un reordenamiento para generar un nuevo espacio urbano, con sustitución total o parcial de los sistemas generales, del espacio edificado, e introducción de nuevos usos con un aprovechamiento constructivo más alto, generando el espacio público requerido» y la reactivación a los «... sectores donde se requiere la habilitación y mejoramiento parcial del espacio público con sustitución parcial y paulatina del espacio edificado. Incluye

acueducto y alcantarillado (...)». Citado en el artículo $4^{\circ}$ - Objeto - del Acuerdo 6 de 1995 «Por el cual se define la naturaleza jurídica de la empresa de acueducto y alcantarillado de Bogotá, E.S.P. y se dictan otras disposiciones».

11 Así como con la Resolución 1190 de 1997, la empresa dispone de criterios para el tratamiento de la población a desplazar.

12 En Bogotá desde hace varias décadas se vienen desarrollando procesos de renovación urbana. Es por esto que en la Ley 9 de 1989 se encuentra el artículo 39 que define los planes de renovación urbana como: «... aquellos dirigidos a introducir modificaciones sustanciales al uso de la tierra y de las construcciones, para detener los procesos de deterioro físico y ambiental de los centros urbanos, a fin de lograr, entre otros, el mejoramiento del nivel de vida de los moradores de las áreas de renovación, el aprovechamiento intensivo de la infraestructura establecida de servicios, la densificación racional de áreas para vivienda y servicios, la descongestión del tráfico urbano o la conveniente rehabilitación de los bienes históricos y culturales, todo con miras a una utilización más eficiente de los inmuebles urbanos y con mayor beneficio para la comunidad» (Ley 9, 1989).

13 Empresa de Renovación Urbana (Acuerdo 33 de 1999).

14 Algunos de los procesos de renovación urbana desarrollados en Bogotá han sido: el proyecto Nueva Santafé, el proyecto de recuperación del barrio Egipto, el reciclaje del edificio conocido hoy como la Calle del Sol, la intervención en el Parque Central Bavaria, el proyecto Ciudad Salitre y el Parque Tercer Milenio, entre otros. 
intensificación en la utilización del suelo y de las condiciones de edificabilidad (ocupación y construcción)» (Decreto 619, 200015.

El reasentamiento por prevención del riesgo (altas condiciones de vulnerabilidad) es asignado a la Fopae y la CVP, e implica la evaluación, seguimiento y control de los factores de riesgo ecológico, promoción de programas para la prevención de desastres, el desarrollo de programas para el saneamiento del medio ambiente, el establecimiento de los costos de deterioro y mantenimiento del medio ambiente, la promoción de investigaciones que permitan identificar elementos críticos y formulación junto con otras entidades de la política nacional de asentamientos urbanos y expansión urbana (Ley 99, 1993). Así mismo, es importante señalar que en 2012 se expide la Ley 1523 por medio de la cual se adopta la política nacional de gestión del riesgo de desastres y se establece el sistema nacional de gestión del riesgo de desastres y se dictan otras disposiciones que son de obligatorio cumplimiento por todos los municipios. Bogotá la incorpora en el Decreto 364 de 2013 que modifica excepcionalmente las normas urbanísticas del POT vigente.

El reasentamiento por desastre (procesos de remoción en masa, inundaciones, fallas geotécnicas y/o geológicas) es coordinado por la Fopae y las acciones posdesastre son atendidas por la CVP, cuyas responsabilidades son precisas en la ley 388 de 1997, cuando se comienza a enriquecer la discusión en torno a la gestión del riesgo. En esta ley son incorporados los preceptos diseñados por el sistema para la prevención y atención de desastres en el ordenamiento municipal. La ley 388 señala que es deber de los municipios

El establecimiento de los mecanismos que permitan al municipio, en ejercicio de su autonomía, promover el ordenamiento de su territorio, el uso equitativo y racional del suelo, la preservación y defensa del patrimonio ecológico y cultural localizado en su ámbito territorial y la prevención de desastres en asentamientos de alto riesgo, así como la ejecución de acciones urbanísticas eficientes (Ley 388, 1997).

En 2011 se dicta la ley 1454, también conocida como LOOT —ley orgánica de ordenamiento territorial_ por la cual se dictan normas orgánicas sobre ordenamiento territorial, sin que los temas de riesgos, vulnerabilidad o reasentamiento estén incorporados en el ordenamiento, a pesar de los impactos generados en los últimos años derivados del cambio climático y la indebida acción del hombre sobre el territorio.

\section{CASOS DE ESTUDIO}

Con el fin de identificar los impactos que ocasiona el traslado de población en los procesos de reasentamiento por riesgo, se estudiaron dos casos que permitieron

15 Este se mantiene igual en el Decreto 190 de 2004, en los artículos 373 a 377 . En el Decreto 364 de 2013 suspendido provisionalmente por el Consejo de Estado el Artículo 379 los reelabora, aunque mantiene los lineamientos básicos planteados desde 2000. 
contrastar las acciones implementadas por las instituciones y los efectos en la población.

El primer caso en la localidad Ciudad Bolívar, zona identificada por la DPAE como de alto riesgo no mitigable por remoción en masa; el segundo caso, un proceso en la localidad de Engativá, en un área que se encontraba dentro de la ronda del río Juan Amarillo, identificada por la $\mathrm{EAAB}$, siendo afectada la población por frecuentes inundaciones.

\section{1. Caso I: Barrio Altos de la Estancia, Ciudad Bolívar}

Localizado en el sector de Altos de la Estancia en la localidad Ciudad Bolívar al sur oriente de Bogotá, en la UPZ 69 Ismael Perdomo. Esta UPZ es catalogada como de urbanización incompleta, con una extensión de 554,9 ha (SDP; 2011a) (figs. 2 y 3$)$.

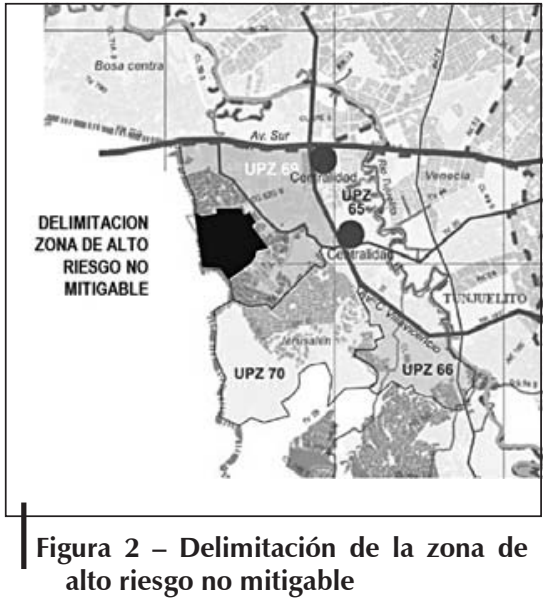

Fuente: Alcaldía mayor de Bogotá $-\mathrm{AMB}-2004 \mathrm{a}$

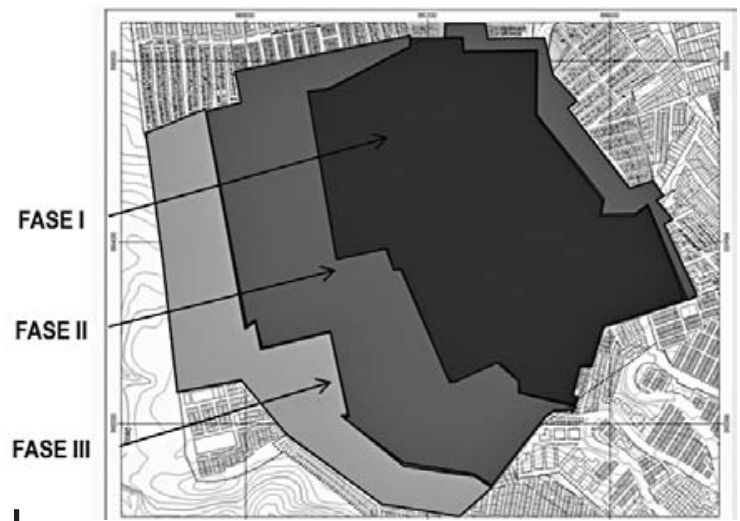

Figura 3 - Fases de Intervención en la zona de alto riesgo no mitigable

Fuente: Alcaldía mayor de Bogotá —AMB - 2004a

En este sector los antecedentes están asociados a cuatro momentos: deslizamiento en 1999 (reasentamiento de 111 familias del barrio Santa Viviana, 44 familias del barrio Espino sector El Rodeo y 168 familias del barrio San Antonio); deslizamiento en 2000 (reasentamiento de 127 familias de Santa Helena y 134 familias de San Antonio); un tercer momento en 2002 se inicia por parte la DPAE16: la delimitación

16 Fondo de Prevención y Atención de Emergencias. La dirección de prevención y atención de emergencias _DPAE - es la oficina gubernamental adscrita a la secretaría de gobierno de la Alcaldía Mayor de Bogotá, que define las políticas e integra las acciones de prevención de riesgos y atención de desastres de las diferentes entidades que conforman el sistema distrital de prevención y atención de emergencias - SDPAE-. 
de la zona de alto riesgo no mitigable y la declaración de suelo de protección por riesgo por parte del DAPD (actual SDP). Para 2003 se evalúa nuevamente el polígono (Fase I) y los sectores aledaños a este, emitiendo los conceptos técnicos que permitieron definir otros dos polígonos para el desarrollo de una segunda y tercera fase de reasentamiento (DPAE, 2003).

Con base a ello se definió el reasentamiento de 3033 familias a cargo de la CVP. Esta tuvo como objetivo garantizar la protección del derecho a la vida de la población localizada en zonas de alto riesgo no mitigable a través de alternativas habitacionales de reposición, legales, seguras y sostenibles, además de contribuir al mejoramiento de la calidad de vida de la población (Fundación Hábitat Colombia, 2005: 10).

Las características que presentaba el asentamiento están asociadas a las condiciones de vulnerabilidad por los riesgos a los que veía expuesto el sector y por las condiciones de pobreza, un alto nivel de hacinamiento en la vivienda, bajo nivel de acceso a servicios públicos y sociales, bajo nivel educativo, falta de fuentes económicas y empleo, precariedad de la construcción, alto nivel de organización comunitaria, y desconocimiento del riesgo (Robles, 2008).

El pago para el traslado de la población se implementa por parte de la CVP con base en el reconocimiento de las mejoras de la vivienda a reasentar, asociado a un esquema financiero que integra cuatro componentes: el valor único de reconocimiento VUR-, el subsidio familiar distrital de vivienda, el crédito hipotecario, y el ahorro programado equivalente al $10 \%$ del costo final de la vivienda.

Sin embargo para 2002, fecha en que se inicia el proceso, no se había reglamentado el VUR y los subsidios para vivienda no dependían ni estaban bajo control de Bogotá. Así mismo, en el proceso no se tiene en cuenta las unidades productivas que se encontraban en el sector delimitado, y de la población incluida en el PRB. Para 2008 se había realizado el traslado voluntario de manera individual de 2068 familias, como se observa en las figuras 4 y 5.

La localización posterior al reasentamiento de la población proveniente de Altos de la Estancia en la ciudad tiene cuatro destinos: la misma localidad, probablemente porque las redes económicas, sociales y culturales tenían mayor consolidación; las localidades que concentran la producción de VIS en la ciudad (Bosa-Usme); otras localidades de Bogotá, probablemente por cercanía con otros familiares; y, la población que con el proceso de reasentamiento fue trasladada a otros departamentos y municipios, evidenciando el desplazamiento y movilización de esta fuera de la ciudad.

La investigación arrojó de manera puntual, en relación a la población después del reasentamiento, los siguientes hallazgos: el 95\% de la población accedió a una alternativa habitacional en condiciones de legalidad (77\% vivienda nueva, 15\% vivienda usada y el 3\% a lotes); el $60 \%$ de las nuevas viviendas (nuevas y usadas) han sufrido modificaciones, debido a que sus espacios no son suficientes para cubrir las necesidades de los hogares reasentados; el 82\% de la población tiene la percepción que en el lugar donde vive hoy mejoraron las condiciones de vida 


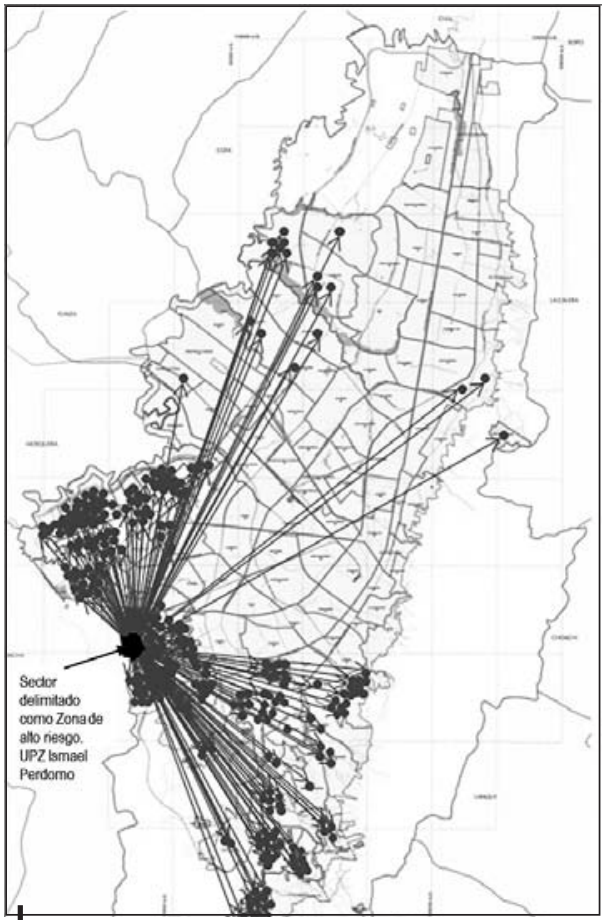

Figura 4 - Destino en Bogotá de la población residente en Altos de la Estancia sujeta del PRB

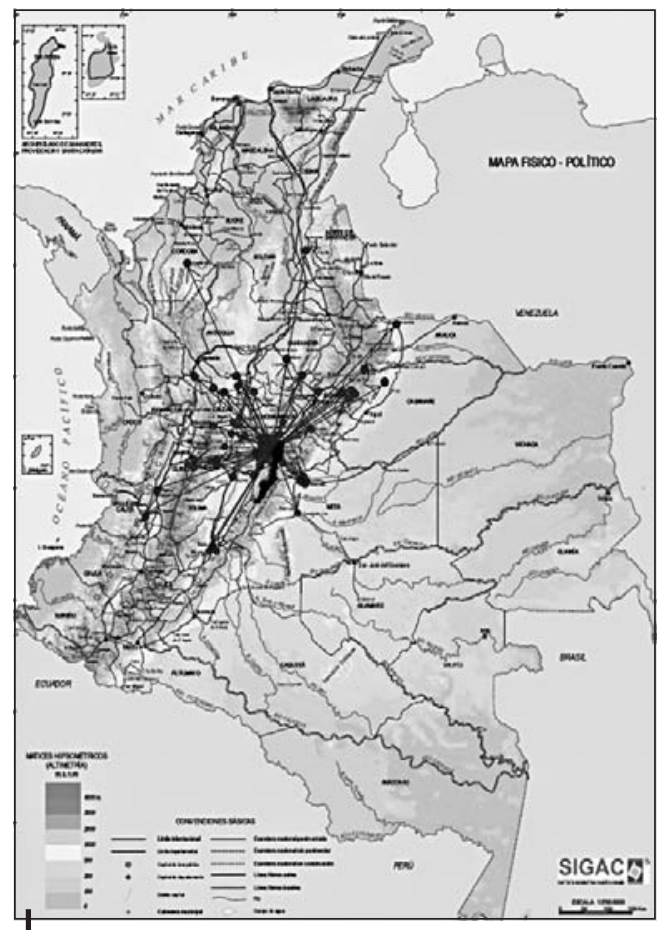

Figura 5 - Destino en Colombia de la población residente en Altos de la Estancia sujeta del PRB

Fuente: Robles, 2008

Fuente: Robles, 2008

de su familia; se presenta un aumento de los gastos que implican la vivienda y los servicios públicos; reconocen un mejoramiento en la calidad y/o acceso a servicios públicos; en cuanto a las actividades económicas aumentaron en comparación con la vivienda anterior. En promedio los gastos de la familia suman $2 \mathrm{SMLV}$, sin embargo los ingresos que reciben son inferiores a estos; en el nuevo asentamiento la población desconoce la existencia de algún tipo de organización comunitaria; el reasentamiento no representó para la población una alternativa colectiva que les permitiera mantener los lazos que tenían previamente como comunidad y por tanto la población no se sintió acompañada durante el proceso de reasentamiento (Robles, 2008).

En este sentido, se puede concluir que el PRB en Altos de la Estancia a pesar de tener resultados positivos en el mejoramiento de la calidad de vida de la población objeto de reasentamiento, está condicionado a la capacidad económica de los hogares para sostener los gastos que implica integrarse a la formalidad, y más cuando la población debe reconstruir no solo las redes económicas, sino las redes sociales y culturales que le permitan mantenerse en los nuevos asentamientos. 


\section{2. Caso II: Barrio Luis Carlos Galán, Engativá}

El barrio Luis Carlos Galán está ubicado en la localidad Engativá al occidente de Bogotá, en la UPZ 29 Minuto de Dios. Esta UPZ es catalogada como de uso residencial consolidado, con una extensión de 367,91 ha, de las cuales 7,91 ha corresponden a suelo protegido y 1,06 ha a áreas sin desarrollar (SDP, 2011b) (figs. 6 y 7$)$.

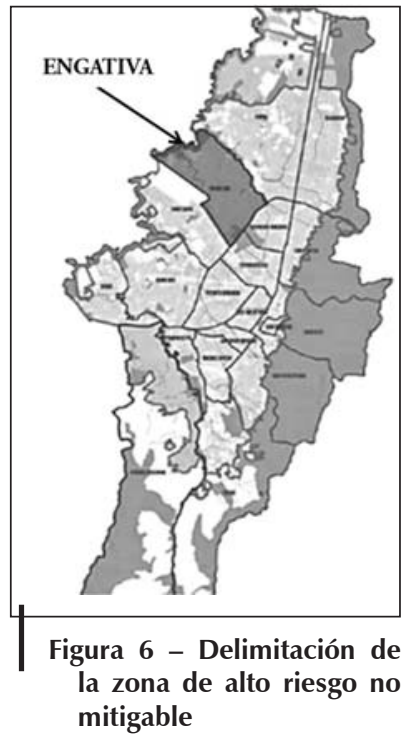

Fuente: AMB, 2004b

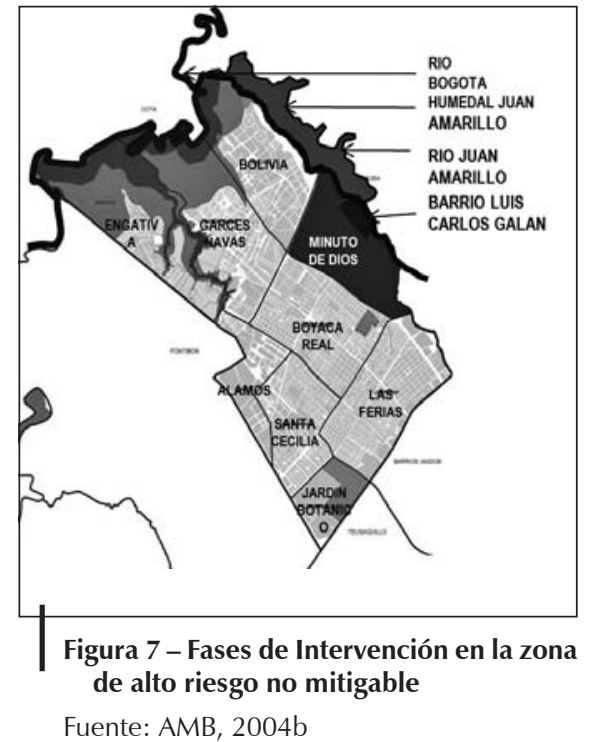

Fuente: AMB, 2004b

En el caso del Barrio Luis Carlos Galán los antecedentes son cuatro: en 1998 la firma Hidrotec Ltda.-Ingenieros Consultores, realizó para la unidad de prevención y atención de emergencias — UPES — de la Alcaldía Mayor de Santa Fe de Bogotá el estudio para la zonificación de riesgos por inundación del río Juan Amarillo y las acciones para su mitigación, estudio que culminó en marzo de 1999. Dentro de esta zona se identificaron las viviendas del barrio Luis Carlos Galán, ubicadas dentro de la ronda del río, por lo que constantemente la población estaba expuesta a inundaciones. En 1999 se contrató una consultoría con el fin de actualizar un estudio socioeconómico que se había desarrollado en 1998, y para la ejecución del reasentamiento. Finalmente se definió el reasentamiento de 226 unidades sociales localizadas en 144 predios de la zona de ronda del río Juan Amarillo.

Las características del asentamiento se pueden sintetizar en que presentaba condiciones de vulnerabilidad por el riesgo; un alto nivel de hacinamiento en la vivienda; bajo nivel de acceso a servicios públicos y sociales; bajos niveles de educación; falta de fuentes económicas y empleo; alto nivel de organización comunitaria, y desconocimiento del riesgo (Robles, 2008). 
Para el traslado de la población se implementa un esquema financiero para sustentar económicamente el reasentamiento bajo la fórmula económica que considera tres aspectos: el subsidio nacional de vivienda, la compensación distrital por las construcciones, y la compensación por actividad económica en el predio. Esta última medida buscaba restablecer las actividades económicas de la población, pero finalmente no se logra, ya que el valor del reconocimiento se integra al valor de la vivienda y no a la restitución de la actividad económica.

En este caso, la población incluida en el PRB se ha trasladado en su totalidad y la reubicación de la población estuvo determinada por tres condiciones de localización: las opciones en la misma localidad con el fin de mantener las redes económicas, sociales y culturales que ya tenían un alto nivel de consolidación; la ubicación de alternativas en las localidades que concentran la producción de VIS en la ciudad (Bosa-Usme); y la localización de la población fuera de la ciudad, en un bajo porcentaje, probablemente por el largo tiempo de residencia que ya llevan en la ciudad (figs. 8 y 9 )

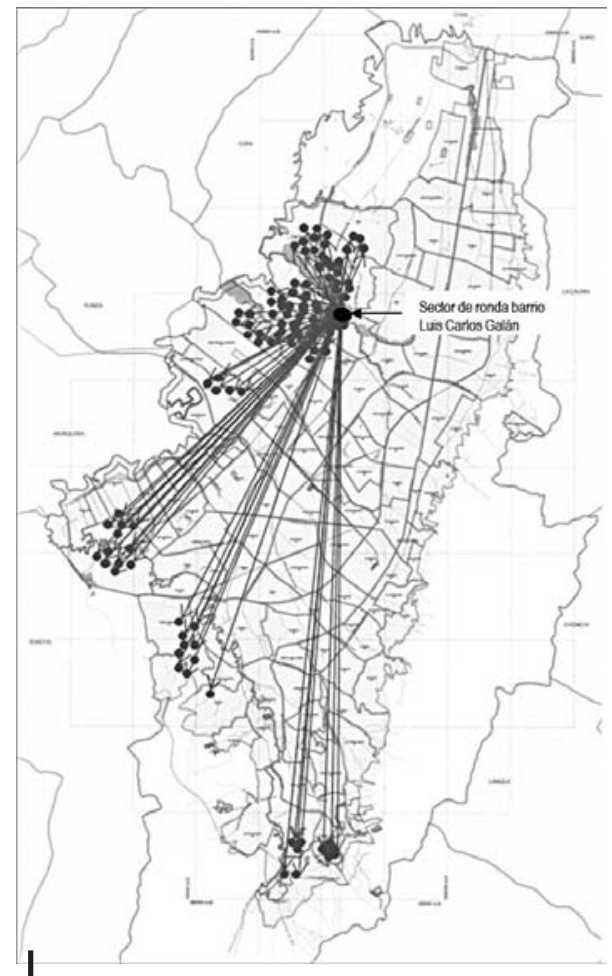

Figura 8 - Destino en la ciudad de la población residente en el barrio Luis Carlos Galán sujeta del PRB

Fuente: Elaboración propia según la Empresa Desarrollo a Escala Humana. En matriz de seguimiento (Robles, 2008)

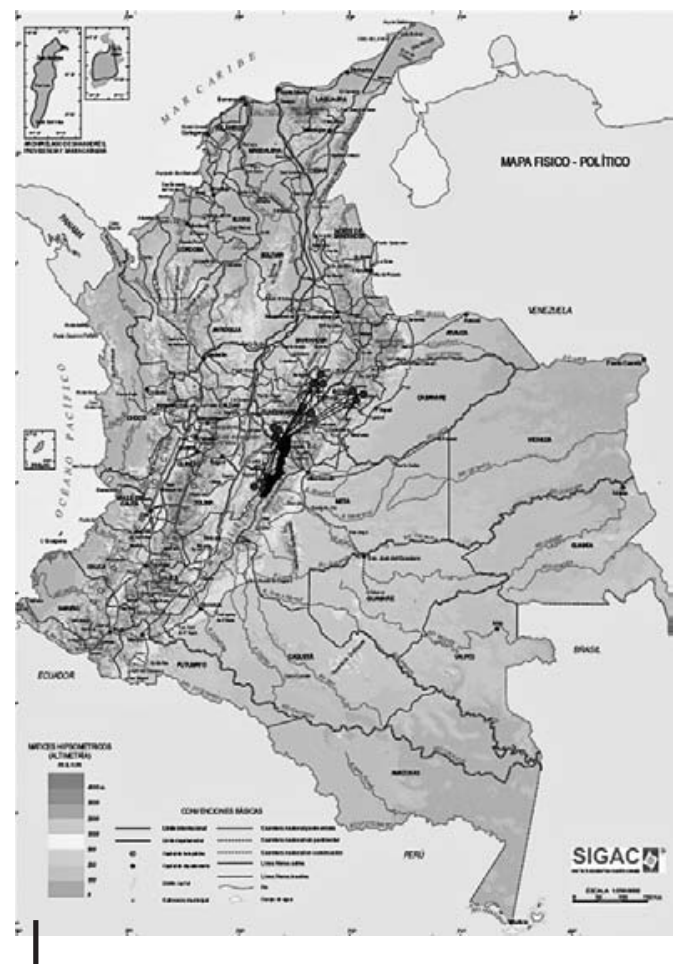

Figura 9 - Destino en Colombia de la población residente en el barrio Luis Carlos Galán sujeta del PRB

Fuente: Elaboración propia según la Empresa Desarrollo a Escala Humana. En matriz de seguimiento (Robles, 2008) 
La investigación, en relación a la situación de la población residente después del reasentamiento, encontró entre otros los elementos siguientes: el 50\% de la población prefirió la compra de vivienda usada, el 36\% prefirió lotes, y el 14\% vivienda nueva; el $75 \%$ de la población tuvo que transformar la nueva vivienda porque los espacios no eran suficientes; el 63\% de la población siente que en el lugar donde vive hoy mejoraron las condiciones de vida de su familia; por el contrario, las actividades económicas disminuyeron en comparación con la vivienda anterior. En este nuevo contexto los gastos de la familia suman aproximadamente $2 \mathrm{SMLV}$, pero los ingresos que reciben son inferiores a este valor. Por otra parte, en el nuevo asentamiento, un alto porcentaje de población reconoce la existencia de organizaciones comunitarias, pero solo el 6\% participa. Así mismo, y al igual que en el caso anterior, el reasentamiento no representó para la población una alternativa colectiva que les permitiera mantener los lazos que tenían previamente como comunidad; de igual modo, la población reasentada desconoce que con el proceso de reasentamiento se realizan paralelamente algún tipo de programa o capacitación (Robles, 2008).

En este sentido, se puede concluir que el PRB en el barrio Luis Carlos Galán, al igual que en el caso anterior, tiene resultados positivos en el mejoramiento de la calidad de vida de la población objeto del reasentamiento, pero así mismo, denota la falta de procesos integrales que aborden otras dimensiones del ser humano más allá de suplir las necesidades físicas de la vivienda, permitiendo a la población incluirse en un nuevo entorno social y económico que garantice su sostenimiento.

A su vez, los hallazgos en estos dos casos de reasentamiento por alto riesgo en Bogotá, permiten señalar que los impactos negativos siguen primando sobre los beneficios esperados para las poblaciones sujeto de reasentamiento. Esta afirmación se sustenta en las grandes limitaciones de dichas intervenciones, que si bien garantizan el reasentamiento no permiten la materialización de la totalidad de derechos que tienen las comunidades, es decir no logra materializarse el derecho a la ciudad.

\section{ALGUNAS CONSIDERACIONES FINALES}

Se puede señalar que los impactos inherentes a los procesos de reasentamiento en Bogotá se tienen técnicamente identificados y se agrupan básicamente en tres: impactos físicos, impactos psicológicos e impactos económicos (Corredor, 2007: 59). Sin embargo, se considera necesario reconocer que los PRB, a pesar de generar impactos negativos, también generan impactos positivos que son los que han potenciado que se sigan realizando este tipo de intervenciones.

De esta manera observamos los impactos relacionados con la materialización del derecho a la ciudad y a la vivienda, las políticas públicas de reasentamiento, y la respuesta institucional, todos ellos a partir del análisis de los componentes físicos, psicológicos y económicos, tanto de orden positivo como negativo.

En el caso de los primeros, los impactos relacionados con la materialización del derecho a la ciudad y a la vivienda, permiten establecer los siguientes elementos 
de orden positivo: a nivel físico, hay un mejoramiento de las condiciones de vida que materializa el derecho a la ciudad para la población que se encontraba en condiciones de vulnerabilidad, y la recuperación de las zonas de riesgo como áreas de protección para la ciudad y la implementación de procesos de prevención para la no ocupación de terrenos no aptos para la construcción; a nivel psicológico, mejoran las condiciones físicas del espacio habitado que se ven representadas en una mejor disposición de la población y de sus capacidades para interactuar en el entorno social. Este mejoramiento se ve también representado en mejores relaciones familiares; y, a nivel económico la mejoría se ve la reglamentación de instrumentos financieros como apoyo para incluir a las familias a un programa de vivienda.

Por otra parte, en los componentes negativos identificados a nivel físico, se ve que, para las familias que no fueron objeto de reasentamiento, el derecho a la ciudad está restringido y la segregación físico espacial se mantiene. Así mismo la ubicación de población en la periferia mantiene problemas de segregación y marginación, así como de gastos y dificultades de movilidad, situación que se complejiza para la población de la tercera edad y en condición de discapacidad, ya que para estas no existe ningún tipo de oferta ni garantía que les permita sostener económicamente la vivienda y adecuarse al espacio. A nivel psicológico, en la mayoría de los casos las condiciones de pobreza se mantienen, lo cual repercute negativamente en el fortalecimiento psicosocial de las comunidades, al igual que la sensación de incapacidad en la población reasentada, al no contar con la información ni las herramientas para defender el conjunto de derechos a los cuales tiene acceso. A nivel económico, la productividad y las condiciones económicas de la población reasentada se ven afectadas a consecuencia del proceso; son mayores los costos de acceso a los servicios públicos y a la movilidad y no se ven amortiguados por ningún tipo de subsidio institucional.

En el caso de los segundos, los impactos positivos y negativos frente a las políticas públicas de reasentamiento se pudieron establecer los siguientes asuntos. A nivel positivo, en materia física se encontró una evolución en la definición del reasentamiento por riesgo en las políticas públicas, así como el reconocimiento del vacío que hay frente a una política específica, también la identificación de la debilidad de las entidades territoriales en la gestión urbana y en la aplicación normativa que incentiva la construcción de políticas propias, al igual que el interés por lograr una articulación de las políticas, programas y proyectos locales con los de nivel nacional. A nivel psicológico, se reconoce la importancia de la inclusión de ámbitos sociales y económicos en los procesos de reasentamiento en los que se contempla la participación comunitaria, la seguridad, la convivencia y la generación de ingresos. A nivel económico, se observa la paulatina inclusión del reasentamiento en la política pública lo que ha permitido el incremento de recursos para su ejecución. En este sentido, se beneficia la construcción de una lógica orientada a la prevención y no ocupación de suelos no aptos para construir, así como la articulación de políticas sectoriales y focalización de recursos del sector público y privado.

A nivel negativo, los aspectos físicos hallados señalan que la inclusión del tema del reasentamiento por riesgo en las políticas públicas aún está muy determinada 
por las construcciones y nociones emanadas de los organismos internacionales, que las soluciones son fragmentadas y son las que recibe la población a causa de la carente gestión urbana, al igual que la baja articulación entre las políticas y las entidades nacionales para el desarrollo de procesos de reasentamiento. En los aspectos psicológicos se presenta la dispersión de la población en tanto es atendida individualmente, lo cual implica el debilitamiento del tejido social y con este de las formas de organización propias y necesarias para la inclusión de estos sectores y de sus intereses en la construcción de políticas públicas. En los aspectos económicos, los subsidios y los créditos no son suficientes y en muchos casos las condiciones de las viviendas a las cuales accede la población no son adecuadas, como tampoco la concentración de oferta de vivienda en el sector privado.

Finalmente y en el tercer caso, a nivel de la respuesta institucional, los hallazgos se pueden señalar de modo positivo indicando a nivel físico la construcción de políticas institucionales para el desarrollo de procesos de reasentamiento que garanticen el mejoramiento de las condiciones de vida de la población; en los aspectos psicológicos, el mejoramiento de las condiciones de vida que repercuten directamente en el fortalecimiento psicosocial de la población; y, en los aspectos económicos, en la construcción de mecanismos financieros para garantizar el acceso de la población a la vivienda. A nivel de los hallazgos negativos encontramos como elementos físicos que la fragmentación de la atención genera la duplicación de acciones o la inasistencia a quienes lo necesitan, así también, la oferta de vivienda institucional en muchos casos no es la adecuada y la población es reasentada en zonas donde se sienten de nuevo en riesgo; de igual modo existe un vacío en la atención social y en el acompañamiento, el seguimiento y control de los procesos de reasentamiento. En los aspectos psicológicos, se identifica el deficiente conocimiento de las condiciones de riesgo por parte de la población, lo que condiciona el desarrollo de las intervenciones, también la ruptura de las redes sociales y culturales de las comunidades y la falta de garantías para que los individuos se inserten en nuevas dinámicas urbanas, así como la ausencia de programas con atención psicosocial que no solamente hagan un monitoreo de las condiciones del reasentado sino que le garanticen la superación de la marginalidad, la exclusión y la segregación en la que se encuentran inmersos. Por su parte, en los asuntos económicos, las condiciones de atención generadas por la lógica del crédito generan distorsiones en la atención de la población, produciendo diferenciaciones y discriminaciones de una población que es evacuada de su hábitat en un mismo momento, pero a la cual se le brinda distintas soluciones en distintos momentos, generando una situación desventajosa para muchas de estas familias; ello genera a nivel económico la débil acción y coordinación de las entidades e implica incurrir en gastos no necesarios.

De este modo, la investigación llama la atención sobre la necesidad de abordar algunos elementos frente a la construcción de la política pública sobre reasentamiento que se constituya en verdaderas estrategias de inclusión de la PBI. Para ello se considera necesario conformar un grupo interinstitucional e interdisciplinario que haga de curador para asegurar que las viviendas se 
entreguen técnicamente construidas y ambientalmente sanas, donde se reconoce que a pesar de existir acciones del Ministerio de Vivienda, Ciudad \& Territorio -MVCT - en este sentido aún son débiles. En el merece especial atención el papel que juegan las curadurías urbanas y su debilidad en las acciones urbanísticas vinculadas con los reasentamientos. A ello se suma la insuficiencia en los procesos de acompañamiento de la población en el transcurso del reasentamiento y en el seguimiento de los impactos que este ocasiona.

Otro asunto clave está relacionado con el papel de las entidades públicas responsables de gestionar y dotar de infraestructuras los suelos para la construcción de VIS, ya que se identifica la necesidad de que estas ejerzan controles en la calidad y habitabilidad de las viviendas, asegurando que la oferta sea adecuada y a un precio justo. Para ello se ha promulgado enorme cantidad de normatividad en el nivel nacional —MVCT_ y Distrital —SDP, SDHT_ sin que ello redunde de manera efectiva en una oferta de calidad.

Por su parte, en los grupos poblacionales que se identifican en condiciones de riesgo, se reconocen condiciones de vulnerabilidad social, principalmente en la población infantil y en los adultos mayores, que no son abordadas durante el proceso. Las acciones provistas por la secretaría distrital de salud —SDS - son insuficientes, y a nivel nacional tanto el Ministerio de Salud como el departamento para la prosperidad social _-DPS — no logran llegar a estos niveles de acción en la escala territorial.

De igual modo, la población en edad productiva presente en los procesos de reasentamiento, requiere de opciones que mejoren las oportunidades laborales, a través de la vinculación a centros de capacitación laboral, fortalecimiento de actividades productivas, talleres de formación de empresas y apalancando financieramente proyectos productivos. Para ello se precisa el apoyo y acción de entidades como el SENA, el MINTIC, el DPS, así como de organismos internacionales.

Finalmente, es necesario reafirmar que los PRB y las acciones con sus pobladores se deben abordar de forma integral, como proceso complejo, donde se busque mejorar las condiciones de vida a través de diferentes programas encaminados a garantizar el acceso a una vivienda adecuada, a un entorno seguro, a la salud, a la educación, a fuentes de empleo y al restablecimiento de las condiciones sociales, económicas y culturales, es decir el restablecimiento pleno de sus derechos como ciudadanos.

Pero para lograr esto, se debe mejorar la capacidad institucional, ya que la responsabilidad de estas, a la fecha, termina en el momento en el que se le entrega la alternativa habitacional a las familias, al asumir que con el traslado, la población resuelve de forma individual factores como el acceso a fuentes de trabajo, salud, educación, entre otros. Sin embargo, en necesario que se realice un seguimiento posterior16 (ex-post), para identificar si esta población mejoró sus condiciones de vida, o si, por el contrario, están en un mayor riesgo de empobrecimiento.

16 En Colombia en los procesos de reasentamiento se han realizado evaluaciones ex-post con recursos de la banca. El Banco Mundial, para el caso de la EAAB, ha financiado diferentes evaluaciones ex- 
Para esto, además de reconocer los impactos negativos que se presentan con el traslado de la población, las falencias en los procesos, en las viviendas y en los programas de protección social, se debe plantear de manera clara el alcance de las intervenciones por parte de las entidades para su efectiva mitigación y para ello en necesario promover la articulación de las políticas a nivel nacional y local.

\section{Siglas}

AMB: Alcaldía mayor de Bogotá

CVP: Caja de vivienda popular

DAPD: Departamento administrativo de planeación distrital

DPAE: Dirección de prevención y atención de emergencias

DPS: Departamento para la prosperidad social

EAAB: Empresa de acueducto de Bogotá

ERU: Empresa de renovación urbana

FOPAE: Fondo de prevención y atención de emergencias

IDU: Instituto de desarrollo urbano

MIB: Mejoramiento integral de barrios

MINTIC: Ministerio de Tecnologías de la información y las comunicaciones

MVCT: Ministerio de Vivienda, Ciudad \& Territorio

PBI: población de bajos ingresos

PNUD: Programa de las naciones unidas para el desarrollo

PRB: programas de reasentamiento barrial

SDA: Secretaría distrital de ambiente

SDHT: Secretaría distrital de hábitat territorial

SDP: Secretaría distrital de planeación

SDS: Secretaría distrital de salud

SENA: Servicio nacional de aprendizaje

SMLV: salarios mínimos legales vigentes

UPES: unidad de prevención y atención de emergencias

UPZ: unidad de planeación zonal

VIS: vivienda de interés social

VUR: valor único de reconocimiento

post de los programas realizados como son, entre otros, el reasentamiento de población localizada en el humedal Juan Amarillo en la localidad de Engativá; el reasentamiento de población localizada en el humedal Jaboque, el reasentamiento de población localizada en el barrio Puerto Amor. 


\section{Referencias Citadas}

ACUERDO 33, 1999 - (Noviembre 10). Por el cual se crea una Empresa Industrial y Comercial del Distrito Capital - Empresa de Renovación Urbana; Bogotá: Consejo de Bogotá.

AMB-SDP, 2013 - Documento Técnico de Soporte del Plan de Ordenamiento Territorial SDP; Bogotá: Alcaldía Mayor de Bogotá D.C.

ARRIETA LORA, D., 2005 - Identificación de estudios y acciones necesarias para la formulación del nivel individual de una política de reasentamiento en el Distrito Capital; Bogotá: Secretaría de Hacienda Distrital - PNUD. Informe final.

BRAND, P. (ed.), 2009 - La ciudad latinoamericana en el siglo XXI, Globalización, neoliberalismo, planeación, 346 pp.; Medellín: Universidad Nacional de Colombia. Facultad de Arquitectura.

CODHES, 2014 - Codhes Informa. De la seguridad a la prosperidad democrática en medio del conflicto. Informe sobre el desplazamiento forzado, conflicto armado y derechos humanos, enero-junio-julio-diciembre de 2013. Boletín Informativo de la Consultoría para los Derechos Humanos y el Desplazamiento, número 78; BogotáQuito, 19 de septiembre de 2011. En: www.codhes.org.

CORREDOR, A., 2007 - El reasentamiento en Bogotá, gestión para la atención del desplazamiento involuntario por obras públicas y de saneamiento ambiental; Bogotá: Universidad Nacional de Colombia. Tesis de posgrado para la Maestría en Hábitat, Estudios en Vivienda.

DECRETO 619, 2000 - Por el cual se adopta el Plan de Ordenamiento Territorial para Santa Fe de Bogotá, Distrito Capital; Bogotá: Alcaldía Mayor de Bogotá D.C.

DPAE, 2003 - Ubicación en zonas de alto riesgo no mitigable en Bogotá; Bogotá: Dirección de Atención y Prevención de Emergencias.

EAAB, 2006 - Manual de Proceso de Reasentamiento de Población; Bogotá: Empresa de acueducto y alcantarillado de Bogotá.

FUNDACIÓN HÁBITAT COLOMBIA, 2005 - Reasentamiento de familias localizadas en alto riesgo no mitigable en el sector de Altos de la Estancia, localidad de Ciudad Bolívar, en Bogotá D.C. In: Foro Iberoamericano y del Caribe sobre Mejores Prácticas; Bogotá.

HATAYA, N. et al., 1994 - Barrio a barrio se construye una ciudad: descentralización y servicios urbanos en Bogotá, 157 pp.; Bogotá: Cinep.

LEY 1454, 2011 - Por la cual se dictan normas orgánicas sobre ordenamiento territorial y se modifican otras disposiciones; Bogotá: Congreso de la República de Colombia.

LEY 1523, 2012 - Por medio de la cual se adopta la política nacional de gestión del riesgo de desastres y se establece el Sistema Nacional de Gestión del Riesgo de Desastres y se dictan otras disposiciones; Bogotá: Congreso de la República de Colombia.

LEY 388, 1997 - Por la cual se modifica la Ley 9 de 1989, y la Ley 3 de 1991 y se dictan otras disposiciones; Bogotá: Congreso de la República de Colombia.

LEY 9, 1989 - Reforma Urbana. Por la cual se dictan normas sobre planes de desarrollo municipal, compraventa y expropiación de bienes y se dictan otras disposiciones; Bogotá: Congreso de la República de Colombia.

LEY 99, 1993 - Por la cual se crea el Ministerio del Medio Ambiente, se reordena el Sector Público encargado de la gestión y conservación del medio ambiente y los recursos 
naturales renovables, se organiza el Sistema Nacional Ambiental, SINA y se dictan otras disposiciones; Bogotá: Congreso de la República de Colombia.

PNUD, 2008 - Bogotá, una apuesta por Colombia: Informe de Desarrollo Humano 2008; Bogotá: PNUD Colombia, Alcaldía Mayor de Bogotá, Acción Social, El Tiempo, ASDI.

ROBLES JOYA, S., 2008 - Impactos del reasentamiento por vulnerabilidad en áreas de alto riesgo en Bogotá. 1991-2005; Bogotá: Universidad Nacional de Colombia. Maestría en Hábitat. Línea de investigación estudios en vivienda.

SDP, 2007 - Segregación socioeconómica en el espacio urbano de Bogotá, D.C.; Bogotá: Alcaldía Mayor de Bogotá D.C., Secretaria Distrital de Planeación. Universidad Nacional de Colombia.

SDP, 2011a - UPZ 69 Ismael Perdomo. Diagnóstico físico y socioeconómico de las localidades de Bogotá. Ciudad Bolívar; Bogotá: Alcaldía Mayor de Bogotá.

SDP, 2011 b - UPZ 29 Minuto de Dios Diagnóstico físico y socioeconómico de las localidades de Bogotá. Engativá; Bogotá: Alcaldía Mayor de Bogotá.

SDP, 2013 - Decreto 364. Modificación excepcional de las normas urbanísticas del Plan de Ordenamiento Territorial de Bogotá D. C. , adoptado mediante Decreto Distrital 619 de 2000, revisado por el Decreto Distrital 469 de 2003 y compilado por el Decreto Distrital 190 de 2004; Bogotá: Alcaldía Mayor de Bogotá D.C.

TORRES TOVAR, C. A., 2005 - Bogotá: simultaneidad de transformaciones y contrastes urbanos. Revista Ciudades, 2005, 9 (1): 105-134; Valladolid, España.

TORRES TOVAR, C. A. (Comp.), 2009a - Ciudad informal colombiana: Barrios construidos por la gente, 316 pp.; Bogotá: Universidad Nacional de Colombia. Facultad de Artes, Grupo de Investigación Procesos Urbanos en Hábitat, Vivienda e Informalidad.

TORRES TOVAR, C. A., 2009b - La vivienda para la población desplazada en Colombia. In: Desplazamiento forzado. ¿Hasta cuándo un estado de cosas inconstitucional? Tomo II; Bogotá: Consultoría para los Derechos Humanos y el Desplazamiento.

TORRES TOVAR, C. A., 2012 - Legalización de barrios: acción de mejora o mecanismo de viabilización fiscal de la ciudad dual. Bulletin de I'Institut Français d'Études Andines, 41 (3): 441- 471; Lima.

TORRES TOVAR, C. A. et al., 2011 - Suelo urbano y vivienda social en Bogotá: la primicia del mercado y el sacrificio del interés general, 1990-2010; Bogotá: Universidad Nacional de Colombia. 